\title{
Nucleon structure studies with the COMPASS experiment at CERN
}

\author{
Stephane Platchkov ${ }^{1, a}$ \\ ${ }^{1}$ IRFU/SPhN, CEA Saclay
}

\begin{abstract}
The COMPASS experiment at CERN uses hadron and lepton beams for nucleon structure studies. Most of the data collected so far with a muon beam and either proton or deuteron polarised target were analysed in terms of longitudinally or transversely polarised parton distribution functions and transverse momentum-dependent distributions in the nucleon. A negative hadron beam is used to perform Drell-Yan measurements. The COMPASS large polarised target gives access to several momentum-dependent singlespin asymmetries and provides a stringent test of the fundamental QCD factorisation assumptions. With positive and negative muon beams COMPASS also studies Generalised Parton Distributions using exclusive deeply virtual Compton scattering and meson production experiments. An overview of the most recent COMPASS results is given. The expected physics outcome of the forthcoming measurements is discussed.
\end{abstract}

\section{Introduction}

The COMPASS fixed-target experiment at CERN [1] uses the SPS secondary and tertiary beams for nucleon structure and spectroscopy studies. The COMPASS hadron spectroscopy programme is covered elsewhere in this conference [2]. In the last few years COMPASS has made detailed studies of the nucleon structure using Deep-Inelastic Scattering (DIS) and Semi-Inclusive DIS (SIDIS). The data were collected with a positive, naturally-polarised muon beam, and either proton or deuteron longitudinally polarised target. An important share of the beam time was dedicated to measurements with a transversely polarised target. The DIS and SIDIS data are mainly used to evaluate the polarised parton distribution functions (PDF) and transverse-momentum dependent (TMD) distributions.

Since 2012 COMPASS has initiated a new physics programme, making full use of its highacceptance experimental setup. The nucleon structure share of this programme comprises two parts. In the first part, a negative hadron beam is used to perform Drell-Yan measurements on polarised nucleon and unpolarised nuclear targets. The Sivers momentum-dependent asymmetry measured in Drell-Yan will be compared with the single-spin asymmetries already measured in SIDIS. The comparison should provide a stringent test of the fundamental QCD factorisation assumptions.

The second part of the new programme is devoted to the study of the Generalised Parton Distributions (GPD). Positive and negative muons beams scattered off an unpolarised proton target will allow measurements of both beam charge and spin sum and difference. The results will provide a determination of the transverse nucleon size as a function of the parton longitudinal momentum.

\footnotetext{
ae-mail: S.Platchkov@cea.fr
} 


\section{Quark contribution to the nucleon spin}

Most of the information [3] about the quark helicity contribution to the spin, $\Delta \Sigma$, comes from deepinelastic scattering (DIS) measurements of polarised leptons on polarised targets. In the recent years COMPASS has measured double-polarisation asymmetries on both proton $\left(\mathrm{NH}_{3}\right)$ and deuteron $\left({ }^{6} \mathrm{LiD}\right)$ targets $[4,5]$. The corresponding proton, $g_{1}^{p}$, and deuteron, $g_{1}^{d}$, spin-dependent structure functions are shown in Fig. 1, together with available data from other experiments. The data are displayed as a function of the four-momentum transfer squared, $Q^{2}$, in different $x=x_{B j}$ intervals. The COMPASS data are particularly important as they cover the low- $x$ domain.
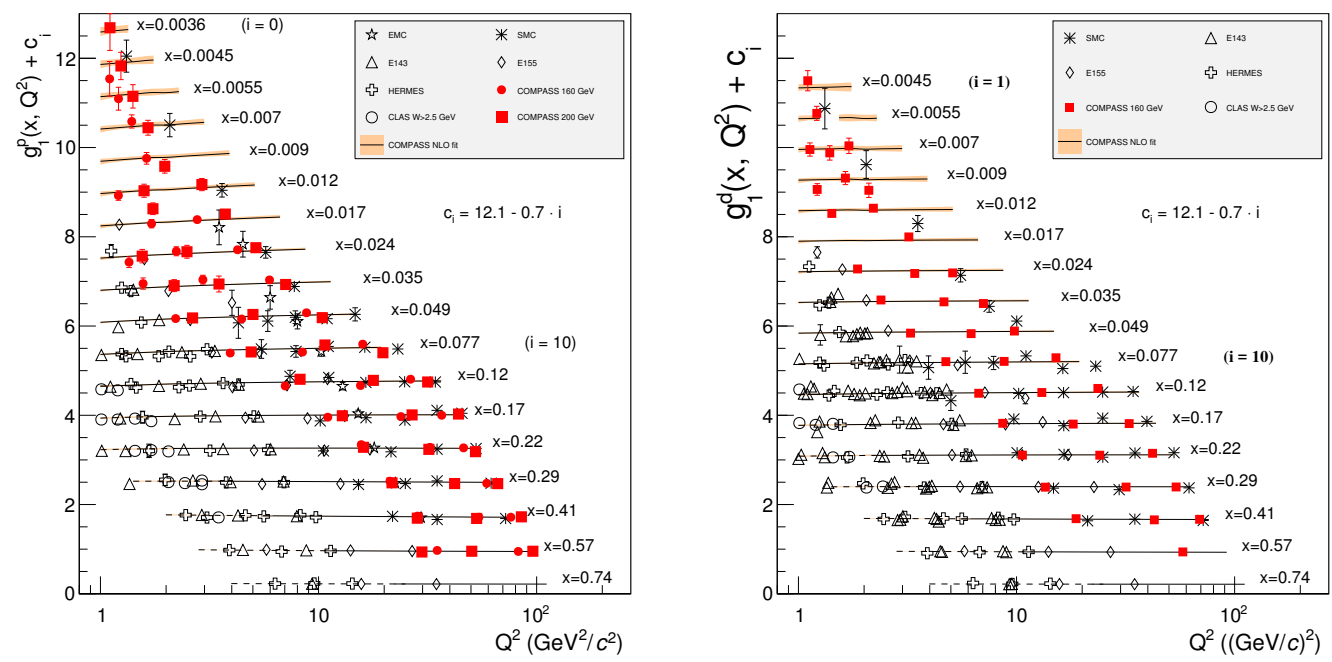

Figure 1. World data on the $g_{1}(x)$ structure function for the proton (left) and for the deuteron (right) a a function of $Q^{2}$, for various x values. The COMPASS data are indicated in red. The lines represent any of the solutions of the COMPASS global NLO QCD fit (see text).

The world data for the spin structure functions for proton, deuteron and neutron targets are included in a global QCD fit [5], performed at NLO. A functional form is assumed at an initial $Q_{0}^{2}$ scale for all singlet, triplet, and octet flavour combinations of the quark distributions, as well as for the gluon distribution. The first moments of the non-singlet distributions are fixed to the values of the axial-vector weak coupling constants measured in neutron and hyperon $\beta$-decay and assuming SU(3) flavour symmetry. The $x-Q^{2}$ evolution ìs done in the $\overline{M S}$ factorisation scheme. In order to investigate the sensitivity of the results to the functional form, the fit was performed for several sets of functional shapes. It was found that two sets of functional shapes are needed to span almost entirely the range of the possible fit results, and particularly for the less-well known gluon contributions, $\Delta g(x)$.

The sets of functional forms, corresponding to the extreme solutions for $\Delta g(x)$, are displayed in Fig. 2. According to each of the solutions, the value of the quark contribution, $\Delta \Sigma$, ranges between 0.26 and 0.34 , or $\Delta \Sigma=0.30 \pm 0.04$. On the top row are shown the quark singlet helicity $\Delta q(x)$ (the integral of which gives $\Delta \Sigma$ ) and the gluon helicity $\Delta g(x)$ distributions. In Fig. 2 bottom row, the quark helicities are shown separately for $\Delta u, \Delta d$ and $\Delta s$ distributions. The solid and dashed lines correspond to two extreme $\Delta g$ solutions of the NLO QCD fit. The dark and light bands correspond respectively 

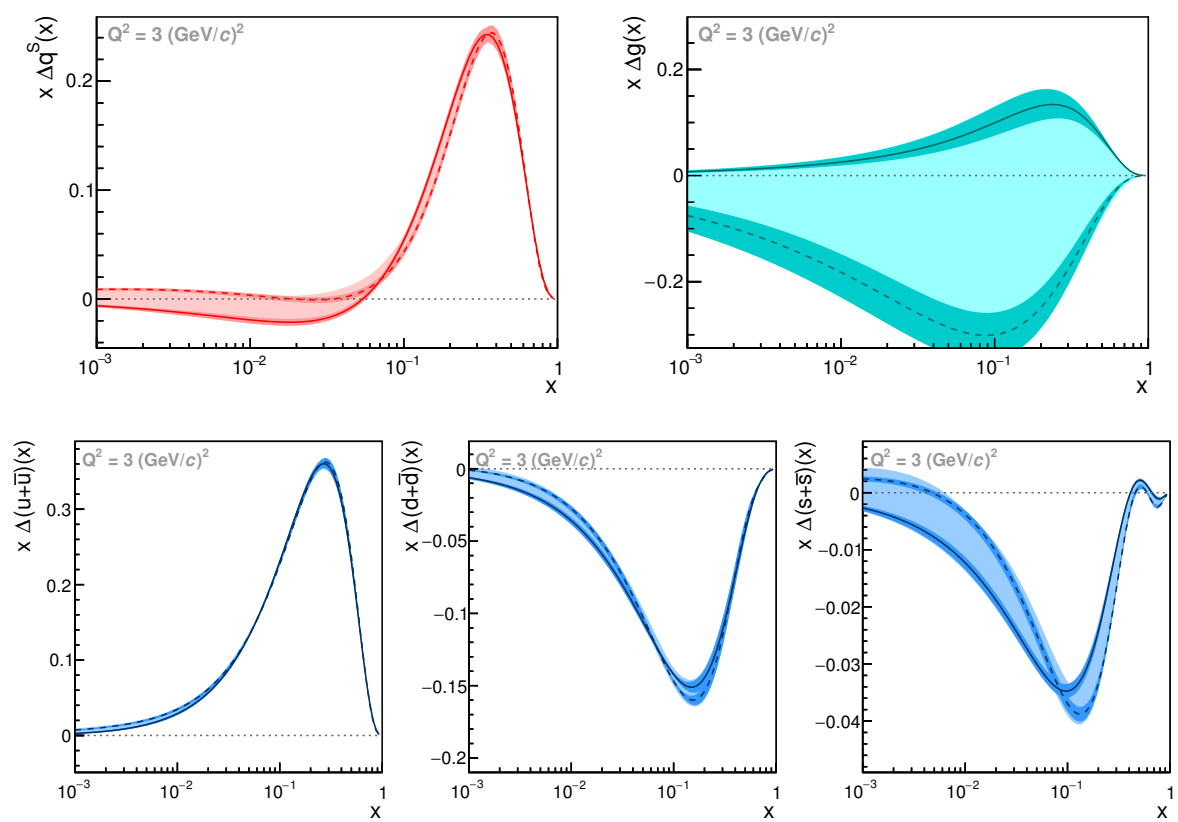

Figure 2. Top: Helicity distributions for quarks (left) and for gluons (right) at $Q^{2}=3(\mathrm{GeV} / c)^{2}$. Bottom left to right: helicity distributions for up, down, and strange quark flavour, respectively. The solid and dashed lines correspond to the two extreme solutions for the $\Delta g$ distributions. The dark bands correspond to the statistical uncertainties and the light bands to the systematic uncertainties.

to the propagation of the statistical errors and the systematic uncertainties. A significant part of the systematic uncertainties is attributed to the choice of the functional form.

\subsection{Gluon contribution to the nucleon spin}

The range of solutions resulting from the different assumptions for the shape of the $\Delta g(\mathrm{x})$ distribution clearly show that the inclusive data are unable to determine neither the shape, nor even the sign of $\Delta g$. Further information on $\Delta g$ is inferred by considering the photon-gluon fusion (PGF) process, in which the virtual photon is absorbed by a gluon in the nucleon, giving rise to a quark-antiquark pair. There are two options for the identification of the PGF process. The first, "open charm" option, is free from physical background at LO. This fact is justified by a negligible quantity of intrinsic-charm in the nucleon [6] in the COMPASS kinematic domain. The open charm is tagged by the invariant mass reconstruction of $\mathrm{D}^{0}$ mesons, via detection of their hadronic decay products [7]. The open charm result for $\Delta g / g$, obtained from $160 \mathrm{GeV} / c$ polarised muon scattering off polarised proton and deuteron targets is shown in Fig. 3.

In the second method, called "high- $p_{T}$ " method, hadron pairs with high transverse momenta $p_{T}$ and $Q^{2}>1(\mathrm{GeV} / c)^{2}$ in the final state are selected [8]. Despite the large statistics available, this method requires a more complicated analysis. Indeed, several processes contribute to the measured asymmetries: the leading order virtual-photon absorption process (LP), the QCD Compton process (QCDC), and the PGF process. In order to extract $\Delta g / g$ from the high- $p_{T}$ asymmetries corresponding 


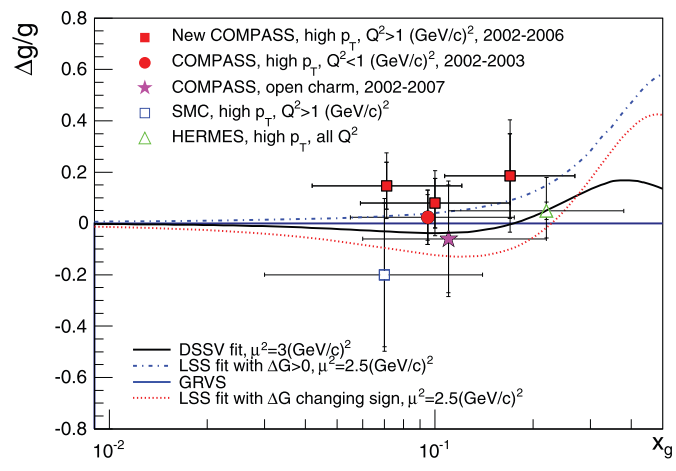

Figure 3. COMPASS results for direct $\Delta g / g$ extraction at $\mathrm{LO}$ using the hadron pairs method (squares) and the open charm method (star). The data points from SMC and HERMES are also displayed. The curves are NLO global fit evaluations of $\Delta g / g$ from Refs [12-14].

to the PGF process only, the contributions from the LP and QCDC processes must be evaluated and subtracted. The corresponding parton asymmetries, as well as the fractions corresponding to each physical process, are determined from a full Monte Carlo simulation of the COMPASS experiment [8]. The resulting data points are shown in Fig. 3. They agree with the open charm point from COMPASS and with the high- $p_{T}$ points from SMC [9] and HERMES [10] experiments, as well as with a previous [11] evaluation by COMPASS using a quasi-real photoproduction process instead of DIS. Figure 3 also shows some of the available world data fits at NLO [12-14].

All COMPASS data on a deuteron target (2002 to 2006) were recently re-analysed, using an extension of the high- $p_{T}$ method, called "all- $p_{T}$ " method [15]. In this new method, the low $p_{T}$ events are kept in the analysis and at least one hadron is required in the final state. The gluon polarization and leading process asymmetry (which is dominant at low $p_{T}$ ) are extracted simultaneously from the same data set using a Neural Network approach. The all- $p_{T}$ method results in a reduction of both systematic and statistical uncertainties by more than $50 \%$, compared to the result of Ref. [8]. The preliminary value of the gluon polarization is $\Delta g / g=0.113 \pm 0.038 \pm 0.035$ at an average gluon momentum fraction $x_{g}=0.10$ and at a scale of $\mu^{2}=3(\mathrm{GeV} / \mathrm{c})^{2}$. All studies indicate that the gluon polarisation is small and either slightly positive or compatible with zero in the $x_{g}$ range of the data.

\subsection{Polarised quark distributions}

The polarised quark distributions for the light flavours are extracted from semi-inclusive DIS (SIDIS) asymmetry measurements. A total of 8 asymmetries are measured [16] at COMPASS, namely $A^{\pi^{+}}$, $A^{\pi^{-}}, A^{K^{+}}$, and $A^{K^{-}}$for both proton and deuteron targets. By also taking into account the two inclusive spin asymmetries, which were used for the extraction of $g_{1}^{d}$ and $g_{1}^{p}$, the $\Delta u(x), \Delta \bar{u}(x), \Delta d(x), \Delta \bar{d}(x)$, and $\Delta s(x)$ quark helicities are evaluated using a LO fit. All helicities are extracted assuming the DSS parametrisation [17] for the quark to pion and quark to kaon fragmentation functions. The results from the extractions are shown in Fig. 4.

The SIDIS results for $\Delta u(x)$ and $\Delta d(x)$ PDFs are compatible with the QCD fit results of the DIS data. However, the strange quark polarised distribution is quite different. The corresponding first moment is: $\Delta s=-0.01 \pm 0.01$ (stat.) \pm 0.01 (syst.). The difference between this result and the one obtained from the inclusive asymmetries, $(\Delta s+\Delta \bar{s})=-0.08 \pm 0.01$ (stat.) \pm 0.02 (syst.), is puzzling.

\subsection{Quark fragmentation functions}

A possible explanation of the $\Delta s$ puzzle could come from the kaon fragmentation functions (FF) used in the SIDIS analysis. Indeed, the extraction of the $\Delta s(x)$ distribution is particularly sensitive [16] 


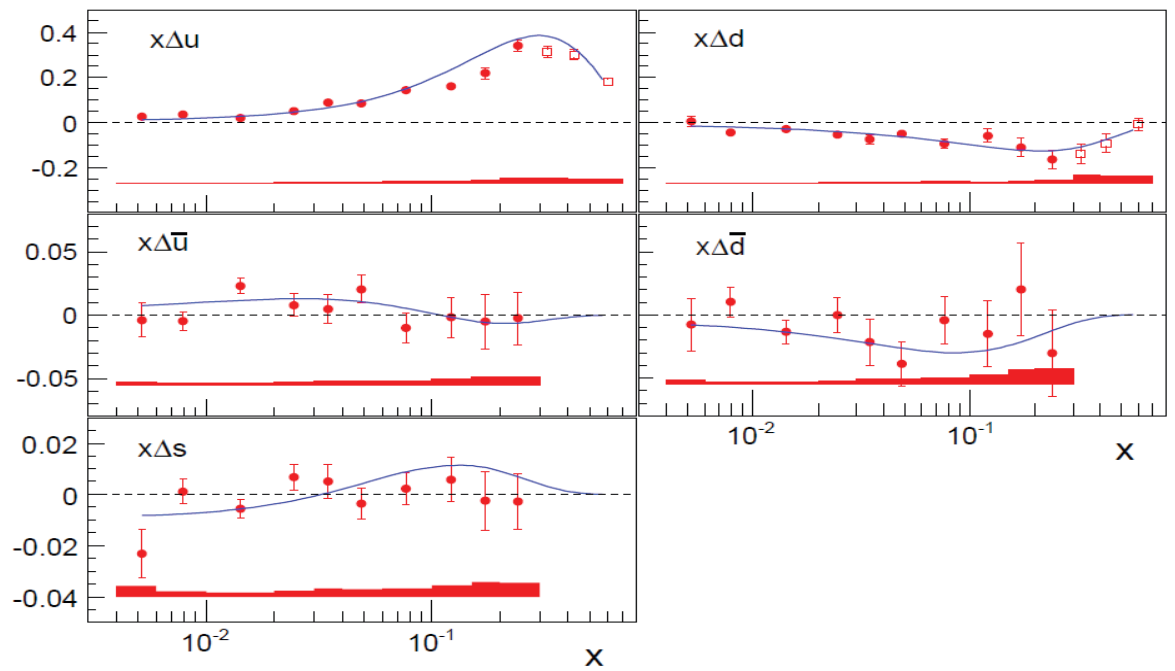

Figure 4. SIDIS results for the light quark helicities, under the assumption that at $Q^{2}=3(\mathrm{GeV} / c)^{2}$. The curves correspond to a global NLO fit taken from Ref. [13].

to the kaon FFs. The FFs are universal and describe the process of hadronisation. They can be extracted via a QCD fit to the pion and kaon multiplicities, which represents the number of produced pions and kaons per deep inelastic events. COMPASS has measured both pion and kaons multiplicities on a deuteron target. Using the $\pi^{+}$and $\pi^{-}$multiplicities data and some additional assumptions (charged conjugation and isospin symmetry), a LO QCD fit is performed. Two pion FFs are fitted: the "favoured" FF (when the quark of the target nucleon is a valence quark of the produced hadron) and the "unfavoured" FF (when the quark of the target nucleon is a sea quark of the produced hadron). Preliminary results for the pion FFs extracted at LO [18] are shown in Fig. 5. The results are compared to the FFs used for analysing the SIDIS results. The new COMPASS FFs are at variance with one
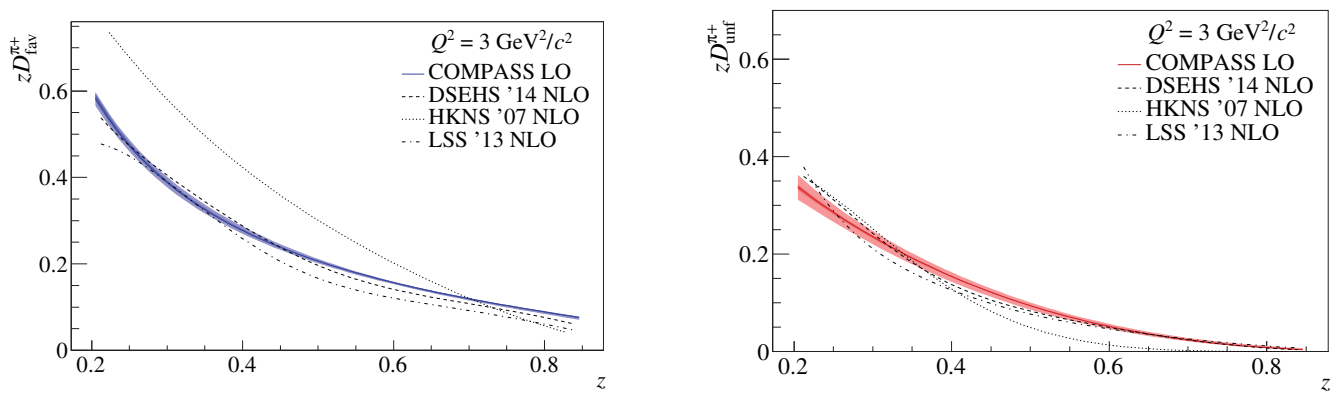

Figure 5. Favoured (left) and unfavoured (right) quark-to-pion FFs from the COMPASS LO fit, compared to the DSS, HKNS and LSS fits at NLO. The light bands represent the correlated systematic and the dark bands the total statistical uncertainties. 
of the older parametrisation [19]. Recent NLO re-analyses [20, 21], which include the preliminary COMPASS pion multiplicities data, are in close agreement with the COMPASS LO fit. The observed differences between the older and new parametrisations of the pion FFs have a minor effect on the extraction of the $\Delta u(x)$ and $\Delta d(x)$ distributions. In connection with the $\Delta s$ puzzle presented above, it is worth noting that the kaon FFs are much less well known than the pion FFs. COMPASS has also measured the kaon multiplicities; preliminary FF fits show large deviations from the available parametrisations.

\section{The three-dimensional spin-dependent structure of the nucleon}

A complete description of the partonic structure of the nucleon in QCD includes the spin and transverse momentum dependent parton distribution functions (TMD PDF). At leading twist there are eight TMD PDFs that take into account the correlations between the quark spin, the quark transverse momentum and the nucleon spin. The TMD PDFs can be accessed in SIDIS by detecting at least one of the produced hadrons. The resulting SIDIS cross section exhibits different azimuthal modulations, with different combinations of two relevant angles: $\phi_{s}$ and $\phi_{h}$. The two angles are the azimuthal angles of the initial nucleon transverse spin vector and the produced hadron momentum, respectively, in

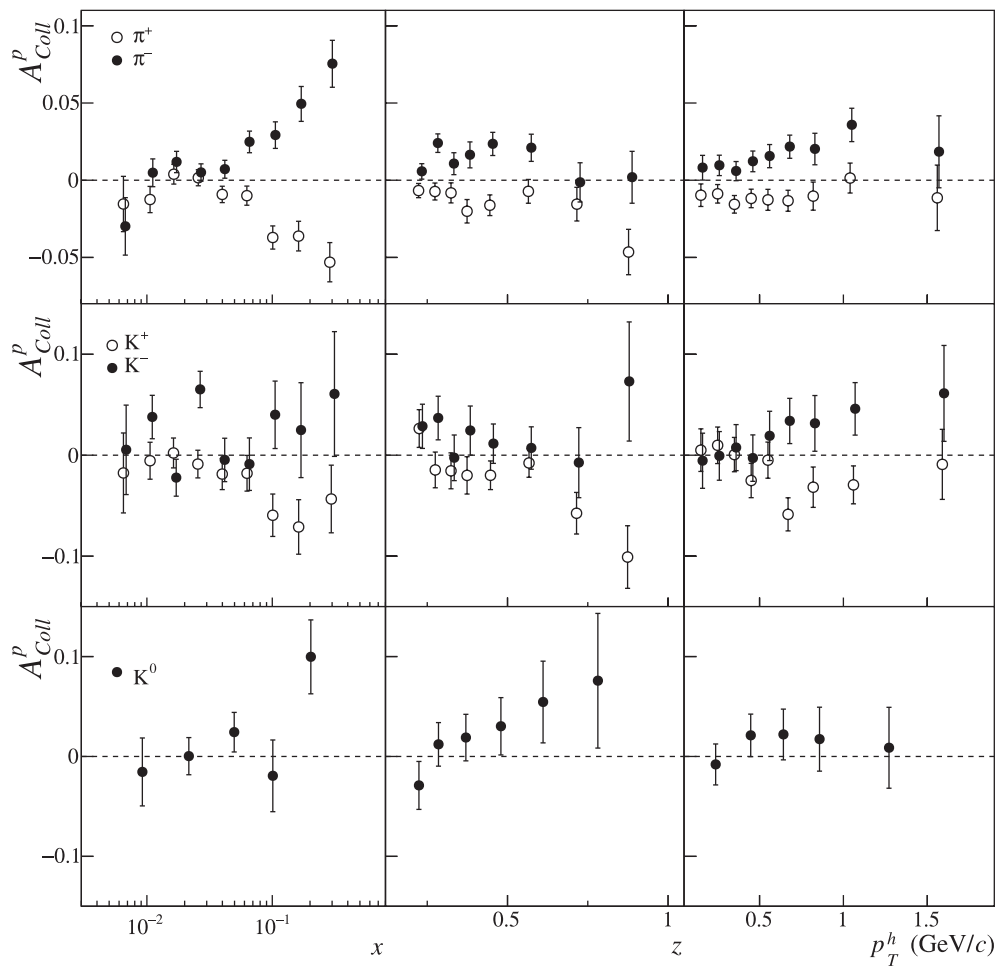

Figure 6. The Collins asymmetries for charged pions (top), charged kaons (middle) and neutral kaons (bottom) as a function of $x, z$ and $p_{T}$. 
a reference system in which the $z$ axis is the virtual photon direction and the $x-z$ plane is the lepton plane according to Ref. [22].

Over the years COMPASS has performed extensive studies of these TMD PDFs using data for single and two hadron SIDIS off unpolarised [23], longitudinally [24] and transversely polarised protons (deuterons) [25] in a $\mathrm{NH}_{3}\left({ }^{6} \mathrm{LiD}\right)$ target. The two most thoroughly studied transverse spin asymmetries are the Collins and Sivers asymmetries.

The Collins asymmetries allow access to the transversity PDF, which is coupled to the Collins fragmentation functions. The results for the Collins asymmetries using a transversely polarised proton target [26] are shown in Fig. 6 as a function of the Bjorken variable, $x$, the fraction of the hadron energy relative to the virtual photon energy, $z$, and the transverse hadron momentum, $p_{T}$. At small $x$ the pion asymmetries are compatible with zero, while in the valence region they show an increasing signal, which has opposite sign for positive and negative pions. The results for charged kaons, although with larger statistical uncertainties, show a similar trend. The Collins asymmetry for neutral kaons shows a positive trend with increasing $z$. The average asymmetry is positive but compatible with zero within the statistical uncertainty.

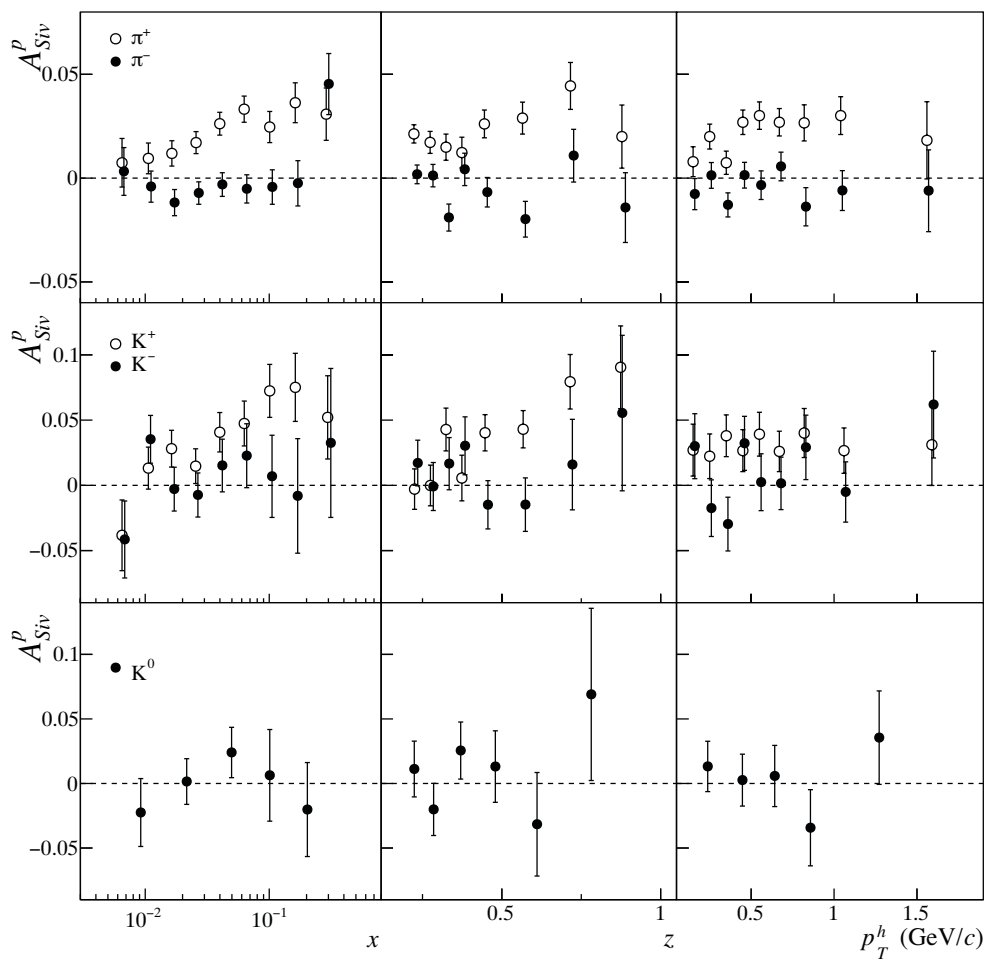

Figure 7. The Sivers asymmetries for charged pions (top), charged kaons (middle) and neutral kaons (bottom) as a function of $x, z$ and $p_{T}$.

Figure 7 shows the COMPASS measurement of the Sivers asymmetries [26] for a transversely polarised proton target, as a function of the $x, z$ and $p_{T}$ variables. The Sivers asymmetries give access 
to the Sivers PDFs, which describe the correlations between quark transverse momentum and nucleon spin. The asymmetries for negative pions and kaons, as well as for neutral kaons are compatible with zero, while for positive pions and kaons there is a clear evidence for a positive signal extending over the full measured $x$ region and increasing with $z$.

The new COMPASS data for the TMD PDFs provide a valuable input for multidimensional global analyses and constitute an important contribution for the understanding of the transverse momentum structure of the nucleon.

\section{Generalised parton distributions}

The Generalised Parton Distributions (GPD) contain additional information on the nucleon structure $[27,28]$. They are accessible at COMPASS via deeply virtual Compton scattering (DVCS) and deeply virtual meson production (DVMP). At leading twist and for a spin 1/2 target, there exist four chiraleven GPDs $(H, \tilde{H}, E, \tilde{E})$ for each quark flavour. The first two of them describe processes that conserve nucleon helicity and the last two those that involve nucleon helicity flip. The GPDs $H$ and $E$ are of special interest as they are related to the total angular momentum carried by the partons in the nucleon [29]. When Fourier-transformed to impact parameter space and for the case of vanishing longitudinal momentum transfer, GPDs provide a three-dimensional description of the nucleon in a momentumcoordinate space, which is also known as "nucleon tomography" [30].

For DVCS on a proton unpolarised target in the COMPASS kinematical domain, the GPD $H$ distribution yields the dominant contribution, while the GPD $E$ is accessible with transverse target polarisation or with a neutron (deuteron) target. All GPDs depend on the photon virtuality $Q^{2}$, the total four-momentum transfer squared, $t$, between the initial and final nucleon states, and two additional
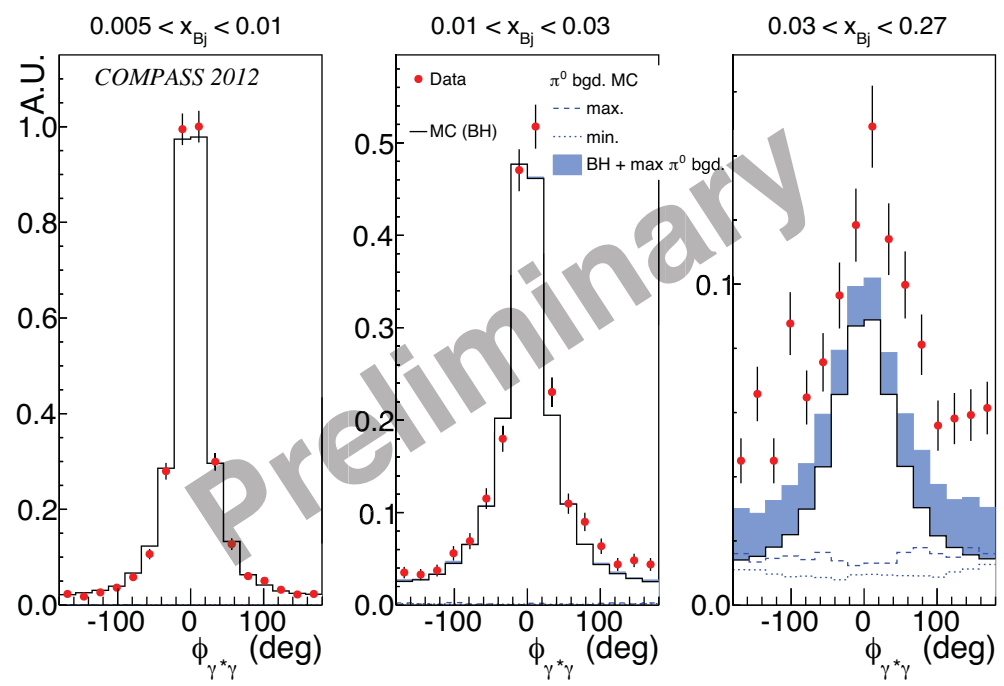

Figure 8. Exclusive single photon events obtained in the 2012 test run as a function of $\phi_{\gamma^{*} \gamma}$ (red dots) compared to the Bethe-Heitler contribution (black line histogram) and the $\pi^{0}$ contamination estimated by a Monte-Carlo simulation. 
variables, $x$ and $\xi$, representing the average and half the difference between the initial and final longitudinal momentum fraction of the nucleon carried by the struck parton. In DVCS and DVMP processes $x$ is an internal variable, while the skewness $\xi$ is related to the Bjorken variable $x_{B j}=Q^{2} / 2 M v$.

The extraction of GPDs from such measurements needs experimental data on hard exclusive processes in a broad kinematic range. Up to now information in the high-energy regime at low $x_{B j}$ was provided by $\mathrm{H} 1$ and ZEUS at DESY. On the other hand, HERMES and JLAB experiments cover the low-energy domain at high $x_{B j}$. The COMPASS measurements will provide a connection between these measurements by covering the region of $x_{B j}$ between 0.01 and 0.1 , where both sea and valence quarks are important.

A competing process is the Bethe-Heitler $(\mathrm{BH})$ process of elastic lepton-nucleon scattering with a hard Bremsstrahlung photon emitted by the incoming or by the outgoing lepton. It produces the same final state as DVCS so that both processes interfere at the level of amplitudes.

In the COMPASS kinematical domain the dominant process is function of the $x_{B j}$ variable. At low $x_{B j}$ almost pure $\mathrm{BH}$ events are recorded; these events provide a reference yield, which is used for normalisation purposes. At higher $x_{B j}$, the DVCS contribution is dominant. After subtraction of the BH contribution, the data can be used for a measurement of the $t$-dependence of the cross section, which provides a tomographic image of the nucleon. In the intermediate $x_{B j}$ domain the DVCS contribution is enhanced by the BH process through their interference. The size of the interference term increases with rising $x_{B j}$ and provides via its characteristic $\phi_{\gamma^{*} \gamma}$ modulation additional possibilities to access real and imaginary part of the complex DVCS amplitude. Here $\phi_{\gamma^{*} \gamma}$ is the azimuthal angle between the leptonic plane (defined by the incident and scattered lepton) and the hadronic plane (defined by the virtual and real photon). The DVCS process itself is dominant for $x_{B j}>0.03$.

The first proof of the feasibility for DVCS was made in 2009 with a reduced experimental setup. In 2012, a four-week long pilot run has been recorded, with a mostly complete DVCS setup, which included a full-scale recoil detector and a large-size hydrogen target. The preliminary results from the pilot run are shown in Fig. 8. The data are displayed in three $x_{B j}$ bins as a function of $\phi_{\gamma^{*} \gamma}$. The data confirm the expected behaviour: in the lowest $x_{B j}$ bin, where the DVCS contribution is negligible, the calculated $\mathrm{BH}$ contribution perfectly agrees with the measurements. The $\pi^{0}$ contamination, when one of the decay photon mimics the high energy DVCS photon and the other photon is not detected, is estimated using a Monte-Carlo simulation.

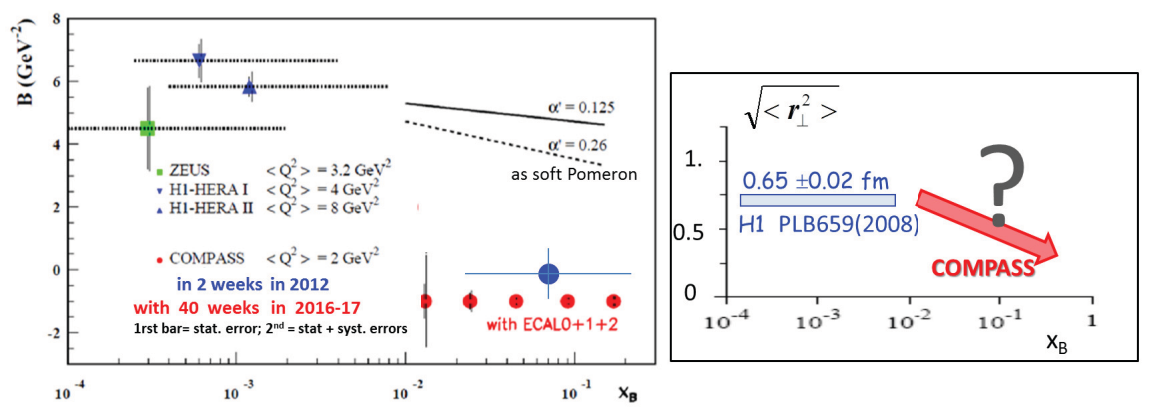

Figure 9. Projections for the measurement of the $x_{B j}$ dependence of the $t$-slope parameter $B\left(x_{B j}\right)$ of the DVCS cross section for a two years data taking, together with the expected measurement from the 2012 pilot run. The data from HERA (see text) are shown as triangles (left). Sketch of the corresponding $x_{B j}$ dependence of the mean proton radius (right). 
The full DVCS run is planned for 2016 and 2017. The data taking will include cross sections measurements with both muon charge and polarisation states of the incoming lepton beam, $d \sigma\left(\mu^{+, \rightarrow}\right)$ and $d \sigma\left(\mu^{-, \leftarrow}\right)$. The real and the imaginary part of the Compton form factors (CFF) can be extracted from the sum $\mathcal{S}$ and the difference $\mathcal{D}$ of these two two cross-sections. A CFF is a sum over flavours $f$ of convolutions of the respective GPDs with a perturbatively calculable kernel describing the hard photon-quark interaction. In the difference $\mathcal{D}$ the pure BH contribution cancels out. The analysis of the $\phi_{\gamma^{*} \gamma}$ dependence provides a measurements of the real part of the corresponding CFF. On the other hand, in $\mathcal{S}$ the BH contribution does not cancel and has to be subtracted. Integrating over $\phi_{\gamma^{*} \gamma}$ yields the $t$ dependence of the cross section, and therefore the transverse size of the nucleon at different values of $x_{B j}$.

Figure 9 left shows the $t$-slope parameter $B$ as a function of $x_{B j}$. In the region of low $x_{B j}$ this parameter has been measured in HERA by H1 [31] and ZEUS [32] to be constant. A decrease is expected at the higher $x_{B j}$ region covered by COMPASS. The projected COMPASS results should clearly measure this decrease. The point with a large horizontal bar represents the statistical uncertainty of the 2012 pilot run measurement. Here, the shrinkage parameter $\alpha^{\prime}$ is related, through a simple relation, to the decrease of the nucleon size with increasing $x_{B j}$. Figure 9 right summarises this information for the proton strong radius, instead of $B$.

\section{Drell-Yan measurements}

In the Drell-Yan process (DY), the annihilation of a quark from the beam with an antiquark from the target (or vice versa) produces, via a virtual time-like photon, a pair of leptons with a large invariant mass [33]. The properties of this lepton pair reflect the distributions of the partons inside the interacting hadrons. The Drell-Yan process thus provides a powerful tool for probing the parton substructure of the hadrons by allowing an extraction of the shape and the flavour dependence of the familiar PDFs in both beam and target hadrons. In specific cases and for polarised beam or target, the Drell-Yan process is also sensitive to the transverse momentum dependent (TMD) parton density distributions.

COMPASS has several unique advantages for performing Drell-Yan studies. First of all, CERN is presently the only place where beams of (positive or negatve) pions, kaons and protons beams are available. The valence antiquarks in the beam interact preferentially with the valence quarks of the target nucleons. Meson-induced Drell-Yan process is the unique way to access the quark structure of pions and kaons, as these short-lived mesons cannot be used as stable targets.

A second advantage lies in the COMPASS experimental setup. The setup, operational since 2002, was especially designed for semi-inclusive and exclusive experiments, requiring detection of several particles in the final state and therefore a large geometrical acceptance. The latter has been achieved by building two consecutive spectrometers around two large-aperture dipole magnets. As a result, the COMPASS acceptance is between 4 and 10 times larger than the acceptances of the previous Drell-Yan experiments. These factors are directly related to the final statistical accuracy achieved. Finally, COMPASS will be able to access the correlation between the nucleon's transverse spin and the transverse momentum distributions of valence quarks by making use of its large-size transversely polarised $\mathrm{NH}_{3}$ target.

The main goal of the COMPASS studies is a measurement of the TMD Sivers asymmetry. QCD factorisation predicts that the Sivers quark distribution function, which depends on the quark transverse momentum, is not universal, i.e. when extracted from the DY process, it should have a sign oppositie to that extracted from a SIDIS reaction. The test of this fundamental prediction is considered as a crucial test of the QCD TMD factorisation.

In order to maximise the statistical accuracy, the relatively low cross-section of the Drell-Yan process is compensated by a high intensity pion beam. A maximum beam intensity achievable in 
COMPASS, close to $10^{8}$ particles per second, is used. A thick hadron absorber is placed immediately downstream the polarised target. In its inner part, the absorber contains a core of tungsten (W) designed to stop the beam which did not interact in the polarised target. This beam plug is in itself a heavy nuclear target. A thin aluminium (Al) target is also placed half-way between the polarised target and the beam plug. The data from the $\mathrm{Al}$ and $\mathrm{W}$ targets will be used for additional unpolarised Drell-Yan studies.
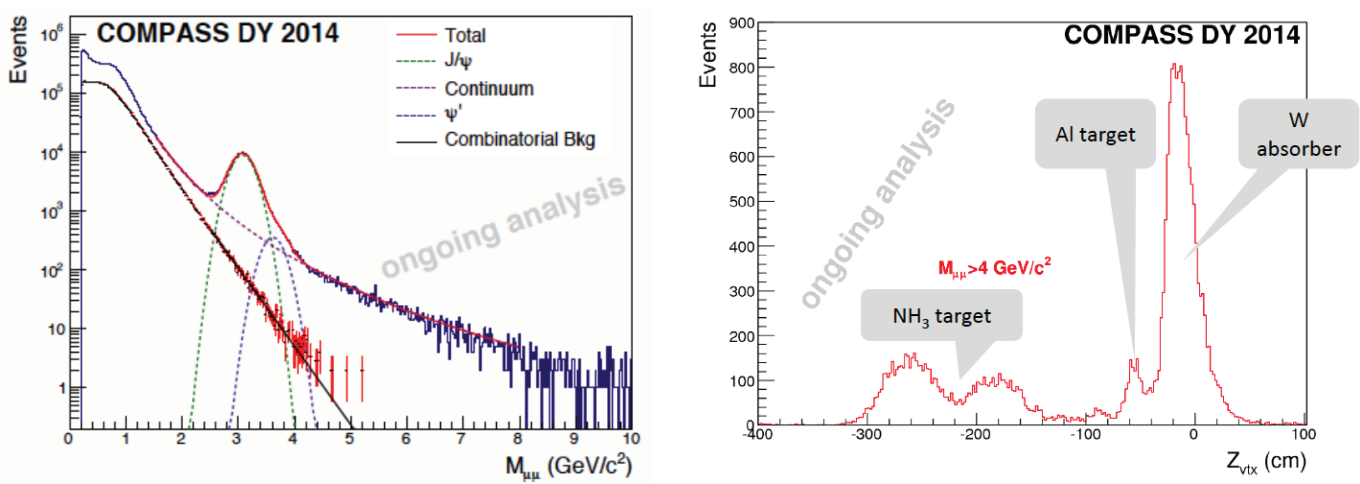

Figure 10. Dimuon mass distribution for two weeks of data taking with an unpolarized target in 2014 (left). Vertex distribution of the events from the three targets in use (right).

A full-scale DY pilot run with the nearly final COMPASS setup, but with an unpolarised target was taken in the fall of 2014. The results from the pilot run are shown in Fig. 10. The resulting mass distribution (left panel) is in good agreement with the expectations, both in terms of mass resolution (estimated by the width of the $\mathrm{J} / \psi$ peak) and counting rates. The reconstructed muon tracks give access to the interaction vertex, as shown in the right panel. The two $\mathrm{NH}_{3}$ cells are well separated and the peaks from the $\mathrm{Al}$ and $\mathrm{W}$ targets are clearly visible. The COMPASS Drell-Yan measurement started in 2015. In the mass range of $4<M_{\mu \mu}<9 \mathrm{GeV} / c^{2}$, about 800 events per day are expected. The expected statistical uncertainty for the Sivers asymmetry from the 2015 data should be of about $2 \%$.

\section{References}

[1] COMPASS Collaboration, P. Abbon et al., Nucl. Inst. Meth. A 577, 455 (2007); COMPASS Collaboration, P. Abbon et al., Nucl. Inst. Meth. A 779, 69 (2015).

[2] F. Nerling, talk at this conference.

[3] C.A. Aidala, S.D. Bass, D. Hasch and G.K. Mallot, Rev. Mod. Phys. 85, 655 (2013).

[4] COMPASS Collaboration, M. G. Alekseev et al., Phys. Lett. B 690, 466 (2010).

[5] COMPASS Collaboration, C. Adolph et al., arXiv:1503.08935.

[6] J. Alwall, PoS, DIS 2005, Madison, USA, arXiv:0508126v1.

[7] COMPASS Collaboration, C. Adolph et al., Phys. Rev. D 87, 052018 (2013).

[8] COMPASS Collaboration, M. G. Alekseev et al., Phys. Lett. B 718, 922 (2013).

[9] SMC Collaboration, B. Adeva et al., Phys. Rev. D 70, 012002 (2004).

[10] HERMES Collaboration, A. Airapetian et al., Journal of High Energy Physics 1008, 130 (2010).

[11] COMPASS Collaboration, E.S. Ageev, et al., Phys. Lett. B 633, 25 (2006). 
[12] E. Leader, A.V. Sidorov and D.B. Stamenov, Phys. Rev. D 82, 114018 (2010);

[13] D. de Florian, R. Sassot, M. Stratmann and W. Vogelsang, Phys. Rev. D 80, 034030 (2009).

[14] M. Gluck, E. Reya, M. Stratmann and W. Vogelsang, Phys. Rev. D 63, 094005 (2001).

[15] M. Stolarski, PoS, DIS 2014, Warsaw, Poland, 211.

[16] COMPASS Collaboration, M. G. Alekseev et al., Phys. Lett. B 693, 227 (2010).

[17] D. de Florian, R. Sassot and M. Stratmann Phys. Rev. D 75, 114010 (2007).

[18] COMPASS Collaboration, C. Adolph et al., to be published.

[19] M. Hirai, S. Kumano, T.-H. Nagai and K. Sudoh, Phys. Rev. D 75, 094009 (2007).

[20] E. Leader, A. Sidorov and D. Stamenov, arXiv:1312.5200.

[21] D. de Florian, et al, Phys. Rev. D 91, 014035 (2015).

[22] A. Bacchetta et al., Phys. Rev. D 70, 117504 (2004).

[23] COMPASS Collaboration, C. Adolph et al., Nucl. Phys. B 886, 1046 (2014).

[24] COMPASS Collaboration, M. G. Alekseev et al., Eur. Phys. J. C 70, 39 (2010).

[25] COMPASS Collaboration, M. G. Alekseev et al., Phys. Lett. B 673, 127 (2009)

[26] COMPASS Collaboration, C. Adolph et al., Phys. Lett. B 744, 250 (2015).

[27] D. Mueller et al., Fortsch. Phys. 42, 101 (1994).

[28] A. V. Radyushkin, Phys. Lett. B380, 417 (1996).

[29] X. D. Ji, Phys. Rev. Lett. 78, 610 (1997).

[30] M.Burkardt, Int. J. Mod. Phys. A 18, 173 (2003).

[31] A. Aktas et al., Eur. Phys. J. C 44, (2005) 1; D. Aaron et al., Phys. Lett. B 659, 796 (2008).

[32] ZEUS Collaboration, S. Chekanov et al., JHEP 0905, 108 (2009).

[33] J.C. Peng and J.-W. Qiu, Prog. Part. Nucl. Phys. 76, 43 (2014).

[34] COMPASS Collaboration, COMPASS-II Proposal, CERN-SPSC-2010-014. 